Original Paper http://ajol.info/index.php/ijbcs http://indexmedicus.afro.who.int

\title{
Prévalence du Banana Bunchy Top Disease (BBTD) dans la zone de Ntoum au Gabon
}

\author{
Jean Alban ONDH-OBAME ${ }^{1 *}$, Auguste NDOUTOUME NDONG ${ }^{1,2}$, \\ Pamphile NGUEMA NDOUTOUMOU ${ }^{2,3}$, Priscilla Chancia MINDZE ASSEMBE ${ }^{1}$, \\ Ignace Davy MENDOUME MINKO ${ }^{1}$ et Kowir PAMBO BELLO ${ }^{1}$
}

${ }^{I}$ Ecole Normale Supérieure de Libreville, Unité d'Enseignement et de Recherche Sciences et Technologies. Département des Sciences de la Vie et de la Terre, Laboratoire LaSciViT. B.P. 17009 ENS Libreville, Gabon.

${ }^{2}$ Centre National de la Recherche Scientifique et Technologique, Institut de Recherches Agronomiques et

Forestières. Département de Phytotechnie, Laboratoire de Biotechnologies Végétales. B.P. 2246 Libreville, Gabon.

${ }^{3}$ Université des Sciences et Techniques de Masuku, Unité de Recherche en Agrobiologie, Institut National Supérieur d'Agronomie et de Biotechnologies, Laboratoire de Biodiversité. B.P. 99 Poto-Poto Franceville, Gabon.

*Auteur correspondant ; E-mail : ondhobame@gmail.com ; Tél. : +241 62286016.

\section{RESUME}

Le Banana Bunchy Top Disease (BBTD) est la maladie virale la plus dévastatrice du bananier impactant considérablement sa production. Le BBTD a été signalé au Gabon pour la première fois par la FAO avec une prévalence de plus de $90 \%$. La présente étude vise à déterminer la prévalence du BBTD, la sévérité et l'importance du vecteur dans la zone de Ntoum. Après une enquête, 1800 pieds de bananiers dans 6 foyers d'infestation, ont été examinés de façon aléatoire avec un système de notation randomisé en transect croisé X. La méthode d'enquête par observation visuelle des symptômes du BBTD avec une échelle de notation de 1 à 5 a été utilisée. Les foyers d'infestation retenus présentent une sévérité de la maladie avancée avec le symptôme visuel de niveau 5 prépondérant et une prévalence moyenne de 21\%. Le vecteur, Pentalonia nigronervosa est un insecte présent dans la zone mais à des niveaux d'importance variable. Il serait souhaitable d'évaluer la résistance variétale des Musa spp. et de montrer l'influence des facteurs biotiques et abiotiques sur la propagation de la maladie.

(C) 2020 International Formulae Group. All rights reserved.

Mots clés : Bananier, Pentalonia nigronervosa, foyers d'infestation, sévérité, importance du vecteur.

\section{Prevalence of Banana Bunchy Top Disease (BBTD) in the Ntoum area in Gabon}

\begin{abstract}
Banana Bunchy Top Disease (BBTD) is the banana's most devastating viral disease, with a significant impact on its production. BBTD was first reported in Gabon by FAO with a prevalence of more than $90 \%$. This study aims to determine the prevalence of BBTD, the severity and importance of the vector in the Ntoum area.
\end{abstract}


After a survey, 1800 feet of banana trees in 6 outbreaks, were examined randomly with a randomized scoring system in transect crossed X. The method of visual observation of BBTD symptoms with a rating scale of 1 to 5 was used. The selected outbreaks have an advanced disease severity with the predominant level 5 visual symptom and an average prevalence of $21 \%$. The vector, Pentalonia nigronervosa is an insect present in the area but at varying levels. It would be desirable to assess the varietal resistance of Musa spp. and to show the influence of biotic and abiotic factors on the spread of the disease.

(C) 2020 International Formulae Group. All rights reserved.

Keywords: Banana, Pentalonia nigronervosa, source of infestation, severity, importance of vector.

\section{INTRODUCTION}

Selon l'Organisation des Nations Unies pour l'Alimentation et l'Agriculture (FAOSTAT, 2017), la banane représente l'un des produits de base de l'alimentation des populations en Afrique centrale avec 35\% d'apports en calories. Elle occupe plus de 50\% de la population active rurale, et contribue significativement au PIB de cette région (FAOSTAT, 2017). Les bananes desserts et les plantains contribuent à la sécurité alimentaire de millions de personnes dans la zone intertropicale du monde et les profits générés par leur culture sont estimés à plus de 50 milliards d'US dollars en 2017.

Le bananier reste vulnérable à de nombreux agents pathogènes (Dhed'a et al., 2001 ; Swennen et Vulysteke, 2001 ; Baudoin et al., 2002), d'origine fongique, bactérienne ou virale (Jones, 2009). L'infection virale la plus dévastatrice pouvant attaquer les bananiers est le Banana Bunchy Top Disease (IITA, 2009; Lepoint et al., 2014). Cette maladie est en pleine expansion dans le monde d'après Kagy et al. (2001), Saghir et al. (2002) puis Kumar et al. (2008 et 2011). Dans de nombreuses zones de cultures bananières, la production et la superficie des bananiers sont réduites de $90-95 \%$ lorsque la maladie est présente (Caruana, 2003). Selon Niyongere et al. (2012), elle est transmise localement, de manière persistante et circulante, par l'agent vecteur Pentalonia nigronervosa Conquerel.

Au Gabon, pays d'Afrique Centrale à très fort potentiel agricole, la maladie du Bunchy Top a été identifiée dans les années 1980 (FAOSTAT, 2018), et jusqu'en 2015, aucun diagnostic n'a été établi. En effet, dans le cadre de la mise en œuvre de la première phase du projet TCP/SFC/3503 «Appui à la prévention et à la gestion de la maladie du Bunchy Top du bananier (BBTD) au Cameroun, en Guinée Equatoriale et au Gabon ", une enquête a été conduite dans les neuf (9) provinces du Gabon (FAOSTAT, 2018). Il en ressort que la maladie est présente dans 8 provinces sur 9 , soit $90 \%$ du territoire national. Cependant, cette étude préliminaire n'a pas considéré le nombre d'individus malades, ni le niveau de sévérité et encore moins l'importance du vecteur. Par contre, la présente étude prend en compte ces principaux critères relatifs à la prévalence de la maladie dans l'optique de déterminer l'occurrence et la distribution du virus du bunchy top et de son vecteur dans la zone de Ntoum.

\section{MATERIEL ET METHODES Cadre de l'étude}

L'étude a été effectuée dans la province de l'Estuaire (Gabon), au sein du département du Komo-Mondah, sur l'axe routier Ntoum Libreville (Figure 1). Le climat est de type équatorial, marqué par deux saisons sèches et deux saisons pluvieuses dans l'année. Selon la classification de Köppen-Geiger, le climat est de type Am. La température moyenne annuelle est de $26,2{ }^{\circ} \mathrm{C}$ et les précipitations annuelles avoisinent $2716 \mathrm{~mm}$, pour une hygrométrie de $98 \%$.

\section{Matériel}

Le matériel végétal de l'étude était issu des différents bananiers des jardins de case et des bananeraies rencontrés tout au long de l'axe routier Ntoum - Libreville. Une diversité de cultivars de Musa spp. a été examinée afin 
d'apprécier la prévalence du BBTD dans la zone.

Un matériel technique diversifié a été utilisé pour l'enquête participative et l'évaluation des différents paramètres à observer. Il s'agit, entre autres :

- du GPS pour relever les coordonnées géographiques des sites ;

- des sacs de congélation pour le transport des échantillons d'insecte ;

- de la glacière pour la conservations du matériel biologique ;

- et du petit outillage agricole pour des travaux divers.

\section{Méthodes}

\section{Echantillonnage}

Les sites d'étude, divisés en six foyers d'infestation et leurs coordonnées géographiques sont: Bikélé $\left(0^{\circ} 23^{\prime} 35,444 \mathrm{~N}\right.$ $62 \mathrm{E})$, Essassa $\left(0^{\circ} 22{ }^{\prime} 21^{\prime \prime} 5 \mathrm{~N}, 9^{\circ} 30^{\prime} 58^{\prime}\right.$ ', $\left.02 \mathrm{E}\right)$,

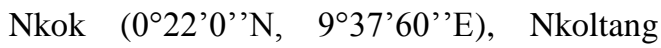
$\left(0^{\circ} 22^{\prime} 2^{\prime},, 24 \mathrm{~N}, 9^{\circ} 38^{\prime} 26^{\prime},, 4 \mathrm{E}\right)$, Okolassi $\left(0^{\circ} 23^{\prime} 45^{\prime},, 96 \mathrm{~N}, 9^{\circ} 36^{\prime} 29^{\prime},, 8 \mathrm{E}\right)$ et Ntoum (0²3'59,92'”N, 9॰27'22', 68E).

Dans chaque foyer d'infestation, 300 pieds de bananiers ont été examinés de façon aléatoire avec un système de notation randomisé en transect croisé $\mathrm{X}$. Chaque ligne du transect représente la moitié de l'ensemble des plants observés sur un site donné. Les 6 foyers d'infestation sont formés à partir des différents sites visités (Tableau 1). En fonction de la superficie de la bananeraie et/ou du jardin de case, des transects de 75,100 et 150 pieds sont examinés respectivement avec des lignes de 38,50 et 75 pieds.

La collecte des données s'est effectuée du 15 juin 2018 au 20 Octobre 2018. Cet intervalle de temps couvre partiellement la grande saison sèche et la petite saison de pluies.

\section{Paramètres observés}

La méthode d'observation visuelle des symptômes du BBTD pour la détermination de la sévérité de la maladie et l'importance du vecteur a été requise, selon les prescriptions de Niyongere et al. (2012). Elle consiste à examiner les pieds de bananiers sélectionnés de façon aléatoire (avec un système de notation randomisé en transect croisé $\mathrm{X}$ ), en se basant sur l'échelle de notation des symptômes visuels et l'échelle de notation de l'importance du vecteur.

Au total, 1800 pieds de bananiers ont été analysés minutieusement. Les observations sur les pieds sélectionnés et analysés portent sur les paramètres suivants :

> La prévalence de la maladie dans la zone, exprimée en pourcentage. Elle est déterminée à partir du nombre de plants infestés au moment de l'enquête sur le nombre total de plants examinés.

$>$ La sévérité de la maladie sur le pied de bananier infecté suivant l'échelle de notation de BBTD de 1 à 5 (Tableau 2);

> L'importance du vecteur sur les pieds observés suivant l'échelle de notation du vecteur de 1 à 5 (Tableau 3).

\section{Corrélation entre la sévérité et l'importance du vecteur}

La corrélation entre la cotation de la sévérité de la maladie sur le pied de bananier infecté suivant l'échelle de BBTD de 1-5 et l'importance du vecteur sur les pieds examinés suivant l'échelle de notation du vecteur de 1-5 consiste en l'étude de la liaison entre la sévérité du BBTD et l'importance du vecteur du BBTV. L'examen de cette question a été fait au moyen d'une analyse factorielle des correspondances (AFC).

\section{Analyse statistique}

Les données ont été soumises à des analyses statistiques. Le logiciel Excel a permis d'obtenir différents graphes illustrant les niveaux de sévérité et l'importance du vecteur à partir de leurs tableaux de contingence, en fonction des foyers d'infestation.

Le logiciel R, sous le package Rcommander, a permis de tester les corrélations pour l'analyse des paramètres. Les résultats ont été obtenus au moyen d'une Analyse Factorielle des Correspondances (AFC) sur R. Les variables étant qualitatives, un test de Khi2 a été effectué afin de vérifier l'indépendance entre la sévérité et l'importance du vecteur. 


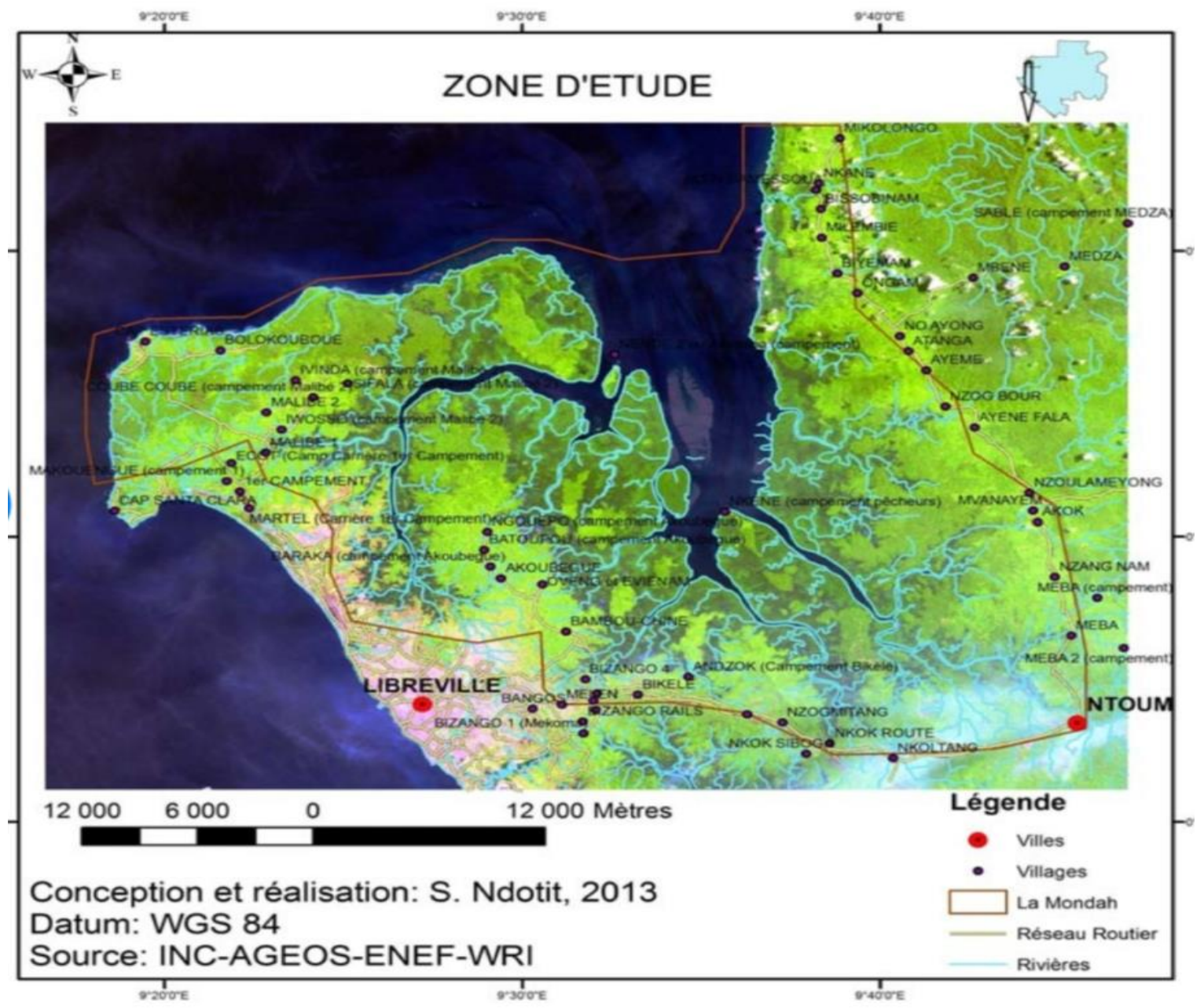

Figure 1 : Délimitation de la zone d'étude (AGEOS, 2013).

Tableau 1 : Subdivision des foyers d'infestation par rapport aux différents sites.

\begin{tabular}{ll}
\hline Foyer d'infestation & Site d'intervention (nombre de pieds observés) \\
\hline Bikélé & PK 15 (150 pieds) - PK 18 (150 pieds) \\
\hline Essassa & Essassa 1 (100 pieds) - Essassa 2 (100 pieds) - Berthe et Jean (100 pieds) \\
\hline Nkok & Nkok 1 (100 pieds) - Nkok 2 (100 pieds) - Nkok-Olam (100 pieds) \\
\hline Nkoltang & Nkoltang 1 (100 pieds) - Nkoltang 2 (100 pieds) - Nkoltang 3 (100 pieds) \\
\hline Okolassi & Okolassi CNSS (150 pieds) - Okolassi 1 (150 pieds) \\
\hline Ntoum & $\begin{array}{l}\text { Ayémé (75 pieds) - Ntoum IGAD (75 pieds) - Ntoum (SEEG) (75 pieds) - } \\
\text { Ntoum Petit village (75 pieds) }\end{array}$ \\
\hline
\end{tabular}


Tableau 2 : Evaluation de la sévérite de la maladie du BBTD grâce aux symptômes visuels selon l'échelle de notation 1 à 5 .

\begin{tabular}{ll}
\hline Niveau & Symptômes \\
\hline Niveau 1 & Stries vertes sur les feuilles \\
\hline Niveau 2 & Stries foncées jusqu'au pseudo-tronc \\
\hline Niveau 3 & Décoloration des feuilles avec taille normale \\
\hline Niveau 4 & Taille réduite des feuilles décolorées \\
\hline Niveau 5 & Aspect touffu au sommet « Bunchy Top » \\
\hline
\end{tabular}

Tableau 3 : Evaluation de l'importance du vecteur du BBTV selon l'échelle de notation 1 à 5.

\begin{tabular}{ll}
\hline Niveau & Caractéristiques \\
\hline Niveau 1 & Une colonie simple sans insectes ailés \\
\hline Niveau 2 & Plusieurs colonies simples \\
\hline Niveau 3 & Une grande colonie avec des insectes ailés \\
\hline Niveau 4 & Plusieurs grandes colonies \\
\hline Niveau 5 & Colonies généralisées au niveau des feuilles et du pseudo-tronc \\
\hline
\end{tabular}

\section{RESULTATS}

\section{Prévalence de la maladie dans la zone d'étude}

L'étude révèle une très forte prévalence dans le foyer d'infestation de la localité de Ntoum (site le plus éloigné de la côte) avec un taux de $38,0 \%$, suivi d'Okolassi $(24,0 \%)$, de Nkoltang $(24,7 \%)$, de Nkok $(18,0 \%)$, d'Essassa (10,3\%) et de Bikélé (11,0\%) qui est plus proche de la zone côtière (Figure 2).

\section{Sévérité de la maladie sur le pied de bananier infecté}

Tous les foyers d'infestation de la zone de Ntoum sont touchés par le BBTD (Figure 3). Le niveau 5 , avec un niveau de sévérité de $36,24 \%$, est le niveau symptomatologique le plus élevé. Il est prépondérant dans la commune, suivi du niveau $4(23,02 \%)$, puis le niveau $3(17,46 \%)$, le niveau $2(12,17 \%)$ et enfin le niveau $1(11,11 \%)$ qui est le niveau le moins élevé et le moins important.
Dans les 6 foyers d'infestation enquêtés, les niveaux des symptômes évoluent tous graduellement selon l'axe d'enquête Bikélé - Ntoum. Le niveau 1 est toujours le niveau symptomatologique le moins rencontré quel que soit le foyer d'infestation (Bikélé $(1,32 \%)$, Essassa $(1,32 \%)$, Nkok $(1,32 \%)$, Nkoltang $(1,85 \%)$, Okolassi $(2,12 \%)$ et Ntoum $(3,17 \%))$.

En revanche, le niveau 5 reste le niveau symptomatologique le plus fréquent quel que soit le foyer d'infestation (Bikélé $(2,38 \%)$, Essassa (2,91\%), Nkok (5,56\%), Nkoltang $(6,61 \%)$, Okolassi $(6,88 \%)$ et Ntoum $(11,90 \%))$. Il en découle une progression significative $(\mathrm{P}<5 \%)$ de la sévérité de la maladie dans l'axe Bikélé - Ntoum, c'est-àdire de la côte vers la zone continentale. La maladie sévit plus dans le foyer de Ntoum $(30,16 \%)$ qui est la zone la plus éloignée de l'océan, où les vents marins sont moins forts. A Ntoum, le niveau symptomatologique 5 
$(11,90 \%)$ y est bien plus important comparativement à tous les autres foyers d'infestation. Les foyers de Bikélé $(2,38 \%)$ et Essassa $(2,91 \%)$, des zones très proches de l'océan sont moins atteintes que tous les 4 autres foyers de l'axe, avec un niveau symptomatologique 5 le plus bas. Sur cet axe, la sévérité de la maladie du BBTD est marquée de façon croissante dans le sens du vent océanique suivant l'axe Bikélé - Ntoum. A l'intérieur du continent, les vents sont moins forts et la sévérité de la maladie est plus importante.

\section{Importance du vecteur sur les bananiers observés}

Le puceron, Pentalonia nigronervosa est présent dans tous les 6 foyers d'infestation de la zone d'étude (Figure 4). Les niveaux 1 et 2 sont quasiment les seuls niveaux d'importance de pucerons présents dans toute la zone, à des proportions variables. Leur présence est graduelle sur l'axe Bikélé Ntoum, où le foyer d'infestation le plus touché est le foyer de Ntoum. La présence des pucerons augmente et devient de plus en plus importante dans la zone à partir des foyers de Nkok, Nkoltang jusqu'à Ntoum. Concernant le niveau 3, il est inexistant dans les zones proches de la côte (Bikélé, Essassa et Okolassi) et n'est présent qu'à Nkok, Nkoltang et Ntoum qui correspondent à des sites éloignés de la côte, avec des vents faibles et en proportion moindre. Le vecteur, Pentalonia nigronervosa, n'atteint pas les niveaux 4 et 5 , peu importe le foyer d'infestation.

\section{Corrélation entre la sévérité et l'importance du vecteur}

Les niveaux d'importance des pucerons 1 et 2 sont prépondérants et leur présence induit l'apparition de tous les niveaux de sévérité (Figure 5).

L'analyse factorielle des correspondances entre la sévérité et l'importance des pucerons fait ressortir deux groupes : le premier groupe est constitué de l'importance des pucerons de niveau 1, associé aux symptômes visuels des niveaux 1,2, 3 et 4 . Le second groupe est constitué de l'importance de pucerons des niveaux 2 et 3 associés aux symptômes visuels de niveau 5 (Figure 6).

Le test de Khi2 a donné une P-value de $5,6896 \mathrm{E}-188$ pour un risque de $5 \%$. Comme 5,6896E-188 est inférieure à 0,05 alors le test est significatif. Statistiquement, il n'y a pas d'indépendance entre la sévérité de la maladie et l'importance du vecteur. Ces deux paramètres sont donc liés.

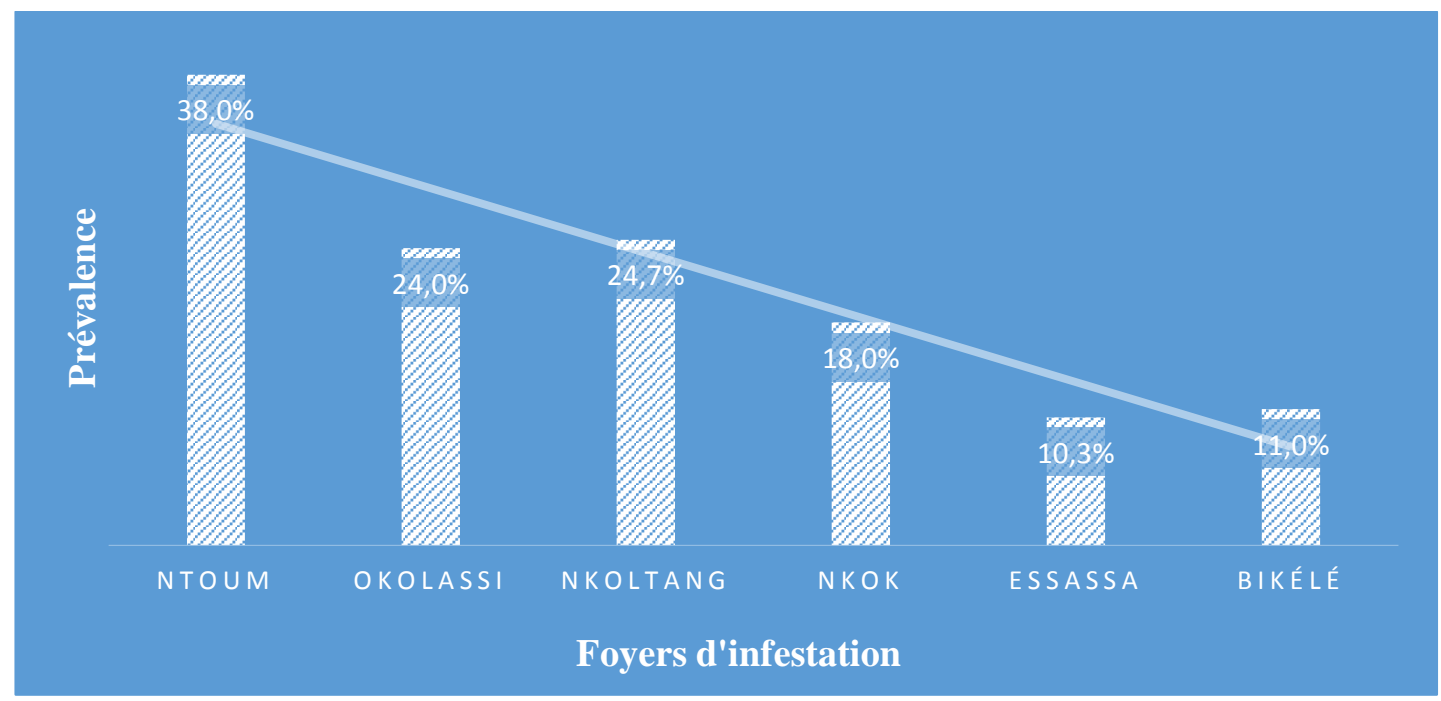

Figure 2 : Prévalence de la maladie du BBTD dans la zone d'étude. 


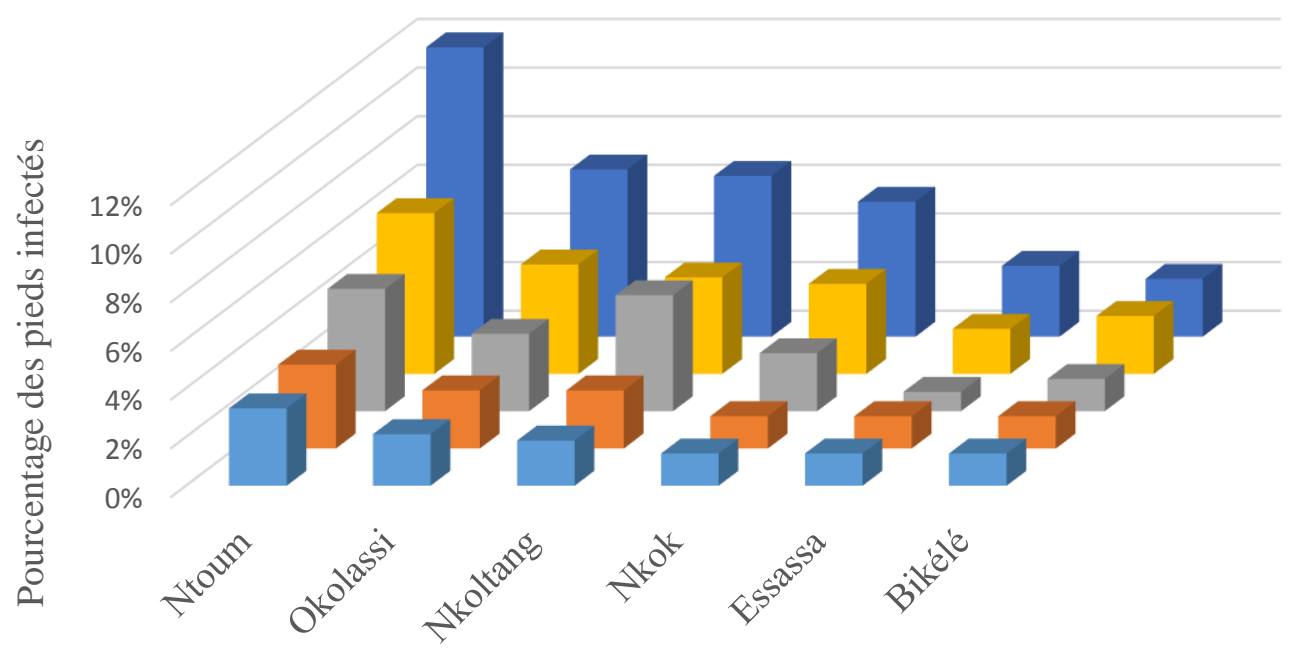

Foyers d'infestation

\section{$\square$ SVN1 $\square$ SVN2 $\square$ SVN3 $\square$ SVN4 $\square$ SVN5}

SVN1 : Symptôme visuel niveau 1= Stries sur les feuilles ; SVN2 : Symptôme visuel niveau 2= Stries vert foncé jusqu'au pseudo-tronc, SVN3 : Symptôme visuel niveau 3= Décoloration des feuilles avec taille normale ; SVN4 : Symptôme visuel 4= Taille réduite des feuilles décolorées ; SVN5 : Symptôme visuel niveau 5= Sommet touffu.

Figure 3 : Sévérité de la maladie du BBTD dans la zone d'étude.

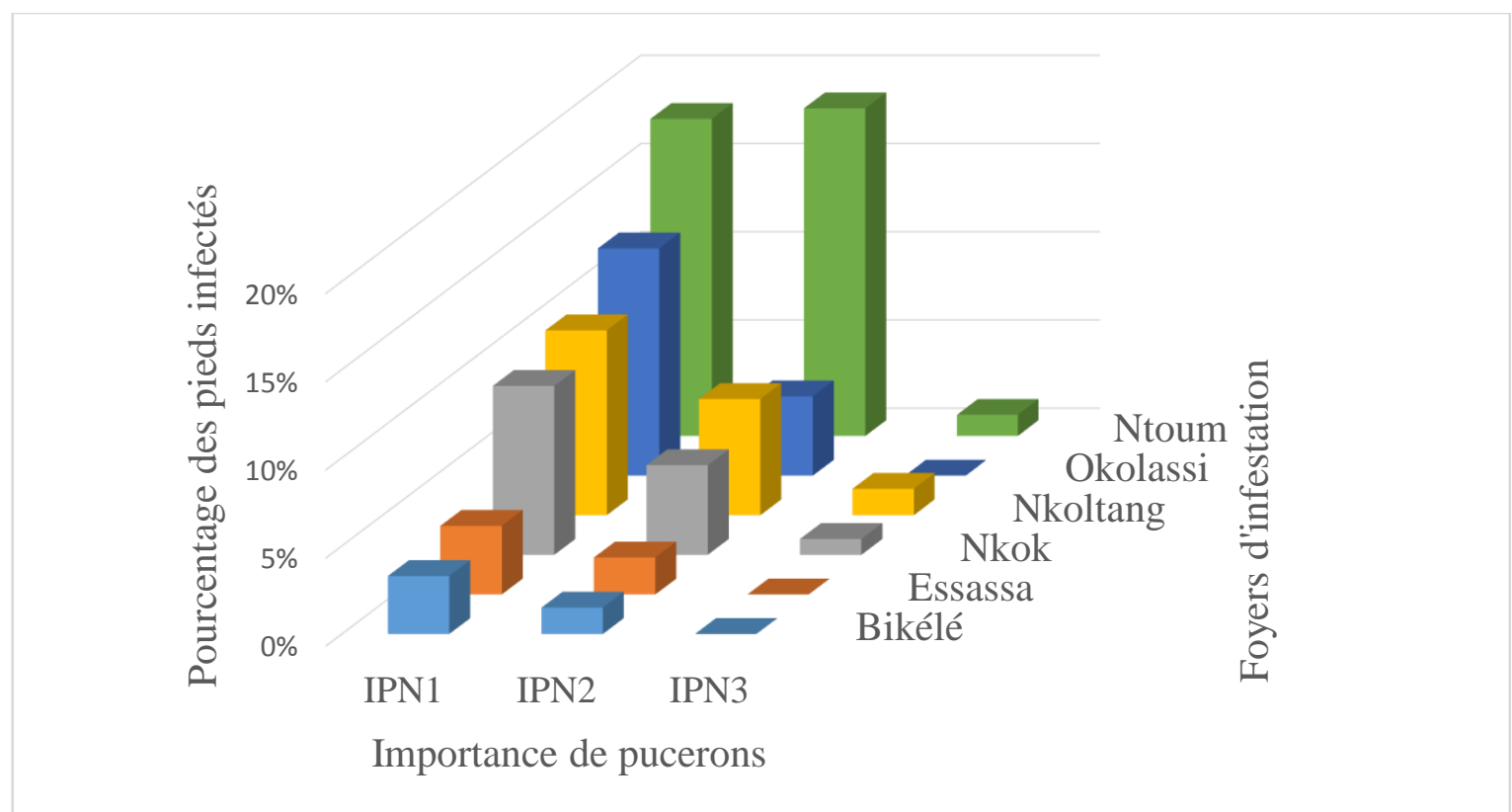

IPN1 : Importance de pucerons niveau 1= une colonie simple sans insectes ailés ; IPN2 : Importance de pucerons niveau 2= plusieurs colonies simples ; IPN3 : Importance de pucerons niveau 3= une grande colonie avec des insectes ailés.

Figure 4 : Importance du puceron vecteur du BBTV dans la zone d'étude. 


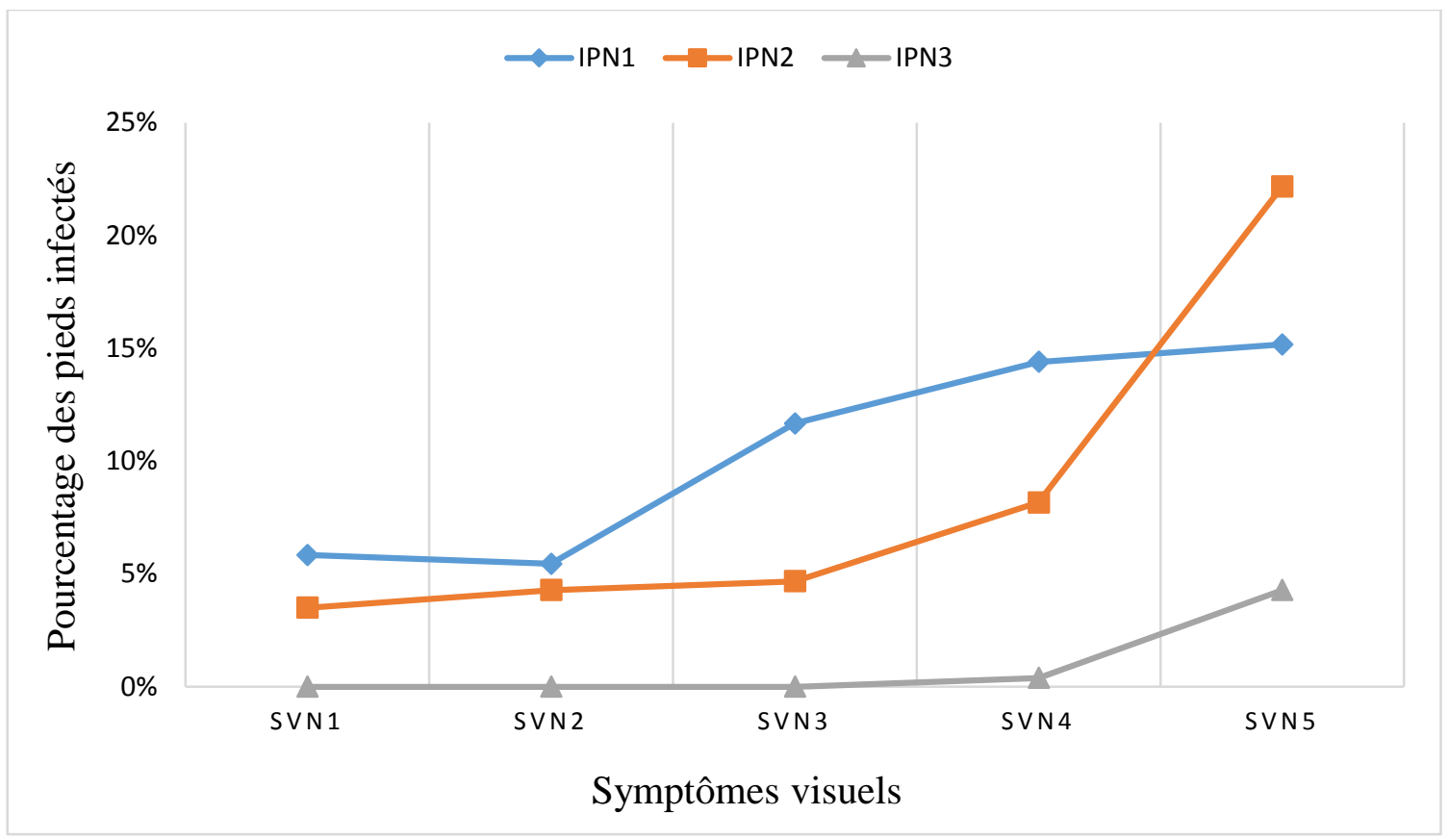

Figure 5 : Corrélation entre la sévérité de la maladie et l'importance du vecteur.

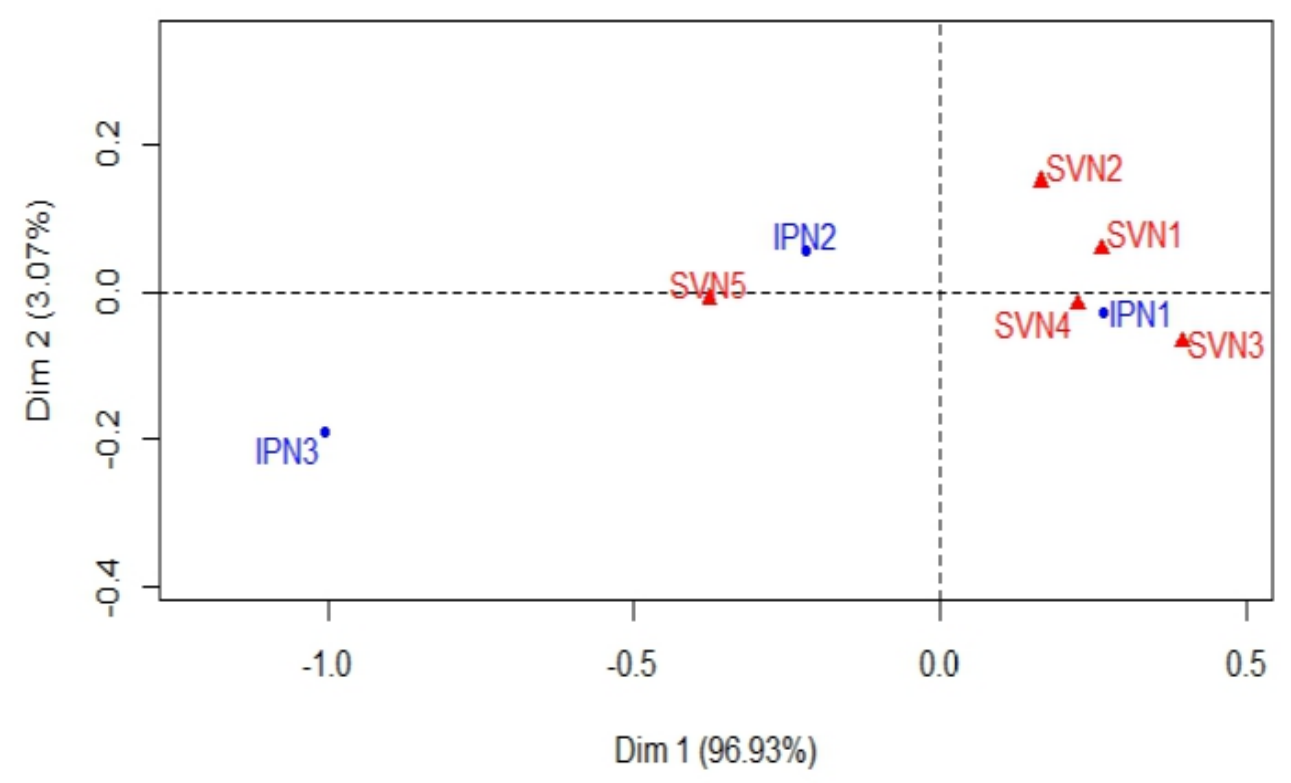

Figure 6 : Analyse en composante principale. 


\section{DISCUSSION}

La forte présence de la maladie dans le foyer de Ntoum peut se justifier par la forte densité du vecteur qui n'y est pas gêné par le vent. Ainsi, les vecteurs peuvent tranquillement inoculer les virus qui vont se répliquer durant une courte période, au point d'inoculation du puceron (Qazi, 2016). Les symptômes apparaissent habituellement sur la seconde feuille émergeante après l'inoculation conformément aux observations de Caruana (2003), puis Busogoro et al. (2009). Cette réplication rapide du virus permet l'apparition immédiate des symptômes au fil du temps. Les niveaux symptomatologiques vont donc évoluer rapidement lorsque les conditions du milieu sont favorables. L'importance des pucerons de niveau 1 peut entrainer l'apparition de tous les autres symptômes visuels du BBTD.

$P$. nigronervosa est la seule espèce d'insecte vecteur connu à ce jour (Thomas et al., 2003). Il a besoin de se nourrir sur une plante infectée pour acquérir des particules virales (Robson et al., 2007 ; Mukwa et al., 2014). Une durée d'alimentation du puceron d'au moins quelques heures sur une plante infectée est nécessaire pour acquérir efficacement le virus. Ce temps est suivi d'une période de latence durant laquelle le virus circule à travers le corps du puceron jusqu'aux glandes salivaires (Adegbola et al., 2013). Le puceron devient ensuite infectieux et capable de transmettre le virus pour le reste de sa vie (Caruana, 2003). Ainsi, quel que soit le nombre de pucerons ou le nombre de colonies de pucerons présent sur la plante, il suffit juste qu'il y ait au moins un puceron vecteur du BBTV pour que la plante manifeste les symptômes de la maladie.

Le symptôme visuel de niveau 5 est toujours associé aux niveaux d'importance de pucerons les plus élevés. En effet, aux températures optimales de $28{ }^{\circ} \mathrm{C}, \quad P$. nigronervosa se reproduit à raison de 25 à 30 générations par an, selon les travaux de Anhalt et Almeida (2008). Les pucerons se localisent généralement en colonies situées entre la gaine de la plus récente feuille jusqu'à la nervure centrale. Les alatae ou formes ailées, apparaissent après 7 à 10 générations d'aptères (sans ailes). D'une manière générale, les ailes apparaissent quand les effectifs à l'intérieur des colonies augmentent fortement, ou lorsque la qualité alimentaire du végétal se détériore (vieillissement ou jaunissement). Cette situation justifie le fait que l'importance de pucerons de niveau 3 n'apparait que lorsque la plante présente les symptômes visuels des niveaux 4 et 5 , en conformité avec les résultats obtenus par Nelson (2004).

La corrélation entre la cotation de la sévérité de la maladie sur le pied de bananier infecté suivant l'échelle de BBTD de 1-5 et l'importance du vecteur sur les pieds examinés suivant l'échelle de notation du vecteur de 1-5 montre bien une liaison entre la sévérité du BBTD et l'importance du vecteur du BBTV. L'analyse factorielle des correspondances (AFC) sur R, dont les résultats sont synthétisés sur la carte AFC montre une correspondance remarquable entre les deux paramètres «Sévérité » et «Importance du vecteur ». Les tests de corrélations et des droites de régressions permettent de comprendre leur corrélation (Yasmin et al., 2001).

Ainsi, les plantes émettent naturellement des phéromones pour se défendre. Ces molécules chimiques vont émettre des signaux qui attirent les pucerons sur un pied de bananier déjà malade. La sève contaminée par le BBTV peut amplifier la reproduction chez les pucerons et donc favoriser leur prolifération. Ce qui justifiera la forte présence des colonies (Importance de puceron niveau 3) sur les plants présentant les symptômes visuels de niveau 5 d'après les travaux de Bayendi (2018) sur les pucerons des cultures maraîchères d'une part, et conformément aux observations de Aka et al. (2009) sur la mosaïque du concombre dans les bananeraies en Côte d'Ivoire d'autre part.

\section{Conclusion}

Il serait souhaitable de mener des études complémentaires dans l'optique de montrer l'influence des facteurs biotiques et abiotiques sur la propagation du BBTD d'une part, et l'importance de la présence du vecteur du 
BBTV d'autre part dans le département du Komo-Mondah au Gabon.

\section{CONFLIT D'INTERETS}

Les auteurs déclarent qu'ils n'ont aucun conflit d'intérêts.

\section{CONTRIBUTIONS DES AUTEURS}

La rédaction principale a été assurée par JAOO, ANN et PNN. L'enquête de terrain et les analyses statistiques ont été menées par JAOO, PCMA, IDMM et KPB.

\section{REFERENCES}

Adegbola RO, Ayodeji O, Awosusi OO, Atiri GI, Kumar PL. 2013. First report of Banana bunchy top virus in banana and plantain (Musa spp.) in Nigeria. Plant Dis., 97 : 290. DOI: 10.1094/PDIS-0812-0745-PDN

Aka AK, Kouassi NK, Agnéroh TA, Amancho NA, Sangaré A. 2009. Distribution et incidence de la mosaïque du concombre (CMV) dans des bananeraies industrielles au Sud-Est de la Côte d'Ivoire. Sciences \& Nature, 6(2): 171-183. DOI: $10.4314 /$ scinat.v6i2.48670

Anhalt MD, Almeida RPP. 2008. Effect of temperature, vector life stage, and plant access period on transmission of Banana bunchy top virus to banana. Phytopatholgy, 98 : $743-748$.

Baudoin JP, Demol J, Louant BP, Marechal R, Mergeai G, Otoul E. 2002. L'amélioration des Plantes. Application aux Principales Espèces Cultivées en Régions Tropicales. Les Presses Agronomiques de Gembloux ; 252p.

Bayendi Loudit SM, Boullis A, Verheggen, Francis F. 2018. Identification of the alarm pheromone of cowpea aphid, and comparison with two other aphididae species. Journal of Insect Science, 18 : 1, DOI: $10.1093 /$ jisesa/iex097

Busogoro JP, Dutrecq O, Jijakli H. 2009. Development of simple molecular protocols to detect Banana bunchy top virus with the PhytoPass System. Acta Horticulturae ISHS, 828 : 213-218.
Caruana ML. 2003. Analyse $d u$ Risque Phytosanitaire (ARP). Bananiers. Banana Bunchy Top babuvivus. CIRAD. P31.

Dhed'a D, Moango M, Swennen R. 2001. La culture des bananiers et bananiers plantains en République Démocratique du Congo, Support didactique, Saint Paul, Kinshasa, 85p.

FAOSTAT. 2017. FAO Regional Office for Africa. La FAO appuie la prévention du Bunchy Top, la maladie du bananier plantain.

FAOSTAT. 2018. Prevention et gestion de la maladie de Bunchy Top du bananier en Afrique centrale.

IITA (International Institute of Tropical Agriculture). 2009. Two diseases could wipe out African bananas, experts step up control efforts. Available online: http://old.iita.org/cms/details/news_detai ls. .aspx ?articleid $=2735 \&$ zoneid $=81$

Jones DR. 2009. Disease and pest constraints to banana production. Acta Hortic., 828: 21-36.

Kagy V, Thomas JE, Sharman M, MadembaSy F. 2001. First record of banana bunchy top disease in New Caledonia. Aust Plant Pathol., 30 : 71.

Kumar PL, Ayodele M, Oben TT, Mahungu NM, Beed F, Coyne D, Londa L, Mutunda MP, Kiala D, Maruthi MN. 2008. First report of Banana bunchy top virus in banana and plantain (Musa spp.) in Angola. Plant Pathol., 58: 402. DOI: https://doi.org/10.1111/j.13653059.2008.01974.x

Kumar PL, Hanna R, Alabi OJ, Soko MM, Oben TT, Vangu GHP, Naidu RA. 2011. Banana bunchy top virus in sub-Saharan Africa: investigations on virus distribution and diversity. Virus Res., 159 : 171-182.

Lepoint P, Staver C, Ajambo S, Lava Kumar P, Hanna R, Caruana ML. 2014. Rapport d'atelier: atelier de planification stratégique 'Reprise de la production bananière dans des zones affectées par la BBTD: Approche au niveau de la communauté et des ménages ruraux. 
Mukwa LFT, Muengula M, Zinga I, Kalonji A, Iskra-Caruana ML, Bragard C. 2014. Occurrence and Distribution of Banana bunchy top virus Related AgroEcosystem in South Western, Democratic Republic of Congo. Am. Journ. Pl. Sci., 5: 647-658.

DOI: http://dx.doi.org/10.4236/ajps.2014 .55079

Nelson SC. 2004. Banana bunchy top; detailed signs and symptoms, College of Tropical Agriculture and Human Resources (UHCTAHR), University of Hawaii. Banana Bunchy Top Virus. Disease spread and development. Available online: http://www.ctahr.hawaii.edu/bbtd/downl oads/BBTV-details.pdf

Niyongere C, Losenge $\mathrm{T}$, Ateka EM, Nkezabahizi D, Blomme G, Lepoint P. 2012. Occurrence and Distribution of Banana Bunchy Top Disease in the Great Lakes Region of Africa. Global Science Books. Tree and Forestry Science and Biotechnology, 6 (1) : 102-107.

Qazi J. 2016. Banana bunchy top virus and the bunchy top disease. J Gen Plant Pathol., 82 (1) : 2-11. DOI: 10.1007/s10327-0150642-7
Robson JD, Wright MG, Almedia RPP. 2007. Biology of Pentalonia nigronervosa (Hemiptera, Aphididae) on banana using different rearing methods. Environ Entomol., 36 : 46-52.

Saghir AR, Mazhar AQ, Abdul LK, Mahammad AK. 2002. Occurrence and incidence of Banana bunchy top disease in southern part of Sindh. Pakistan. Journal of Plant Pathology, 1 (2-4) : 7475. DOI: $10.3923 / p p j .2002 .74 .75$

Swennen R, Vuylsteke D. 2001. Bananier. In Agriculture en Afrique Tropicale, Raemarkers HR (éd.). DGCI: Bruxelles; 611-636.

Thomas JE, Geering ADW, Dahal G, Lockhart BEL, Thottappilly G. 2003. Banana and plaintain. In: Virus and Virus-Like Diseases of Major Crops in Developing Countries, Loebenstein G, Thottappilly G (eds). Kluwer: Dordrecht; 477-496.

Yasmin T, Khalid S, Soomro MH, Malik SA, Shah H, Ahmad I. 2001. Specificity of host-pathogen interaction of banana bunchy top disease. Journ. Biol. Sci, 1(4) : 212-213. DOI: 10.3923/jbs.2001.212.213. 